\title{
A Method for Harmonic Measurement of Real Power Grid Signals with Frequency Drift using Instruments with Internally Generated Reference Frequency
}

\author{
B.M. Antić, Z.L. Mitrović, V.V. Vujičić \\ Department of Power, Electronics and Communication Engineering, Faculty of Technical Sciences, University of Novi Sad, \\ Trg Dositeja Obradovica 6, 21000, Novi Sad, Serbia, antic@uns.ac.rs

\begin{abstract}
The authors have recently developed a number of instruments for measuring harmonic composition of power grid signals. These measured signals equals its nominal value $(50 \mathrm{~Hz}, 60 \mathrm{~Hz}$ or $400 \mathrm{~Hz}$ ). This approach has enabled the measurement of a high number of harmonics within a single period. However, the internal oscillator in the instrument generates the nominal frequency and cannot adapt to frequency changes in the input signal. This paper presents a method for the improvement of the operation of comparison of the measurement error with and without the application of the proposed method is presented in the paper.
\end{abstract} \\ instruments have a simple, predominantly digital architecture and they are based on an assumption that the frequency of the \\ the developed instruments in cases when the fundamental frequency drifts from its nominal value as is the case with real power \\ grid signals. Based on this method, modified versions of the harmonic measurement instruments have been developed. A
}

Keywords: Harmonics, spectral analysis, frequency stability, power grid measurements

\section{INTRODUCTION}

$\mathrm{T}$ HE IMPORTANCE of power quality indicators such as frequency stability, total harmonic distortion factor, signal transients, etc., has been recognized for decades and widely discussed. The economical impact of the power quality concept on the industrial sector [1], [2], and in particular on the performance of power electronic devices [3], and quality of consumer's life [4], has been the subject of long lasting discussions.

The need for more sophisticated control of "polluted" power grids has brought new challenges and restrictions to the measurements [5], [6], which include frequent/continuous low-cost monitoring of the harmonic composition of voltage/current signals in frequency varying environments.

The authors researched the application of the stochastic measurement method in power distribution grid where the measurement interval and the fundamental frequency are strictly defined $(20 \mathrm{~ms} / 50 \mathrm{~Hz}$ in Europe, $16.7 \mathrm{~ms} / 60 \mathrm{~Hz}$ in U.S.).

A typical 3-phase power quality instrument measures 3 voltages $\left(u_{R}, u_{S}, u_{T}\right)$ and 4 currents $\left(i_{0}, i_{R}, i_{S}, i_{T}\right)$ and for each determines the spectral composition in terms of harmonic amplitudes and phases or sine and cosine coefficients up to the order $M$. The order $M$ is usually very low (up to 16), but recently a need has been raised to increase the number of measured harmonics up to 50 or even 100 [7].

One of the key parameters characterizing power quality is the total harmonic distortion factor or THD. The THD is defined with (1) as the ratio of the sum of the powers of all higher harmonic components $P_{2}, P_{3}, P_{4}$, etc., to the power of the harmonic at the fundamental frequency $P_{1}$.

Significant $T H D$ values indicate undesirable situations in power grids such as peak currents, heating, emissions or possible core losses in motors.

$$
T H D_{P}=\frac{\sum_{k=2}^{M} P_{k}}{P_{1}}
$$

In measurements based on harmonic amplitudes the THD can alternatively be expressed as a ratio of RMS values (2).

$$
T H D_{U}=\frac{\sqrt{\sum_{k=2}^{M} U_{k}^{2}}}{U_{1}}
$$

There are two possible approaches to the problem of harmonic amplitude measurements in power grids. The first approach is based on the assumption that the fundamental frequency in power grid is a well-known parameter which never drifts too far from the nominal value. This approach then uses a simple, fast, reliable and inexpensive hardware to perform the measurement. This is the case in almost all applications which rely on the DFT or the FFT and presume unchanged fundamental frequency [8], [9]. When the power grid fundamental frequency differs from the nominal value and the instrument does not adjust to the actual fundamental frequency, an error occurs. The measurement results are no longer signal's harmonics but pseudo-harmonics which contain error both in amplitude and position on the frequency axis. Since the DFT and the FFT suffer from many pit-falls [10], an alternative approach can be considered, which takes into account the unknown fundamental frequency and uses more complex processing of time or frequency samples to obtain the true spectrum of the signal. While this alternative approach is far more advanced, it requests much more expensive and complex hardware with demanding signal processing capabilities. Many such solutions have been developed based on direct measurements in the frequency domain [11], the usage of 
wavelets [12], application of other specific methodologies such as transient analysis [9] and usage of thermal converters [13].

The stochastic measurement method described in [14] and [15], and especially its modification described in [16] is used to obtain coefficients of the trigonometric polynomial expansion of a continuous voltage or current signal over an arbitrary interval using signal dithering. These measurements are characterized with a high accuracy and represent a direct application of the Weierstrass Approximation Theorem [17], which is a special case of more general Stone-Weierstrass Theorem [18]. The dithering technique is widely used to enhance the performance of analogue-to-digital converters (ADC) and to correct their quantization error [19].

In this paper we will focus on the stable regime in power grids which occurs most of the time and which is the basic assumption for power quality measurements. We will also take into account the fact that in reality the fundamental frequency drifts from the nominal value. However, we will show that even with an instrument which incorrectly assumes the fundamental frequency to be equal to the nominal value and performs measurements of pseudo-harmonics, it is still possible to obtain the exact signal spectrum using the incorrect measurement results under a certain set of conditions. These conditions refer to the actual frequency drift and the number of harmonics which are required at the instrument's output. The proposed correction algorithm is far less complex, more reliable and faster than any other known approach based on the assumption of an unknown frequency. A partial solution to the problem can be found in case of a purely sinusoidal signal [20] but the solution cannot be generalized to the nonsinusoidal regime.

The method described in this paper is based on a novel result in discovering a very special feature of the considered problem: the matrix, which maps the vector of actual harmonics into the vector of pseudo-harmonics obtained by the measurement, has a tendency to cumulate around the main diagonal. This feature enables us to apply an extremely efficient algorithm for on-the-fly solving large systems of linear equations. The method can be used to completely eliminate the error caused by frequency variations and the computation can be easily performed within a single period of the input signal (before the results for the next period are available). The range of applicability is extremely broad and covers nearly all cases of harmonic measurements that are of interest. The method requires only a simple hardware upgrade and requires low processing power, so it can easily be implemented into the existing instruments.

\section{SUBJECT \& METHODS}

When measuring harmonics, power quality analyzers normally presume that the voltage and current signals are periodic signals with the fundamental frequency equal to the nominal frequency $f_{0}$ and the corresponding minimal period $T_{0}$. In reality the fundamental frequency varies with changes in power consumption although it remains close to $f_{0}$ as shown in [21]. Thus, real signals are actually not periodic but almost periodic and therefore do not possess a standard
Fourier representation. Fortunately, these changes of frequency could be considered as slow enough. Thus, we can presume that during the interval within which the measurement has to be performed the signals remain periodic with a fundamental frequency $f$ which has drifted from the nominal (expected) value $f_{0}$ for an amount $\Delta f_{0}$. To represent such an almost periodic signal with a given/assumed error $\varepsilon$ we use the well-known Weierstrass trigonometric polynomial approximation [17]:

$$
y(t)=\frac{a_{0}}{2}+\sum_{k=1}^{M}\left(a_{k} \cos 2 \pi f k t+b_{k} \sin 2 \pi f k t\right)
$$

where $a_{n}$ and $b_{n}$ are coefficients of this polynomial and $M$ is the order of the highest harmonic corresponding to the choice of $\varepsilon$.

Assuming that the DC component is not present in a signal defined with (3), the THD given with (2) can also be calculated using (4):

$$
T H D_{U}=\frac{\sqrt{\sum_{k=2}^{M}\left(a_{k}^{2}+b_{k}^{2}\right)}}{\sqrt{a_{1}^{2}+b_{1}^{2}}}
$$

To take into account the varying frequency problem, let

$$
T=\frac{1}{f}=\frac{1}{f_{0}+\Delta f_{0}} \neq T_{0}
$$

be the minimal period corresponding to the actual fundamental frequency $f$. According to [17], $a_{n}$ and $b_{n}$ coincide with Fourier's coefficients of the signal $y(t)$ and could be expressed in the following integral form

$$
\begin{aligned}
& a_{n}=\frac{2}{T} \int_{0}^{T} y(t) \cos \frac{2 \pi n t}{T} d t, n=0, \ldots, M \\
& b_{n}=\frac{2}{T} \int_{0}^{T} y(t) \sin \frac{2 \pi n t}{T} d t, n=0, \ldots, M
\end{aligned}
$$

However, a non-adaptive instrument which measures signal $y(t)$ with the period $T$ assuming that $T$ is equal to $T_{0}$ will generate pseudo-harmonics:

$$
\begin{aligned}
& \hat{a}_{n}=\frac{2}{T_{0}} \int_{0}^{T_{0}} y(t) \cos \frac{2 \pi n t}{T_{0}} d t, n=0, \ldots, M \\
& \hat{b}_{n}=\frac{2}{T_{0}} \int_{0}^{T_{0}} y(t) \sin \frac{2 \pi n t}{T_{0}} d t, n=0, \ldots, M
\end{aligned}
$$

The principal schematic of such an instrument that measures 3 voltages and 4 currents is shown in Fig.1. Note that this instrument has an internal counter which generates reference frequency of $50 \mathrm{~Hz}(20 \mathrm{~ms}$ period) [16]. 


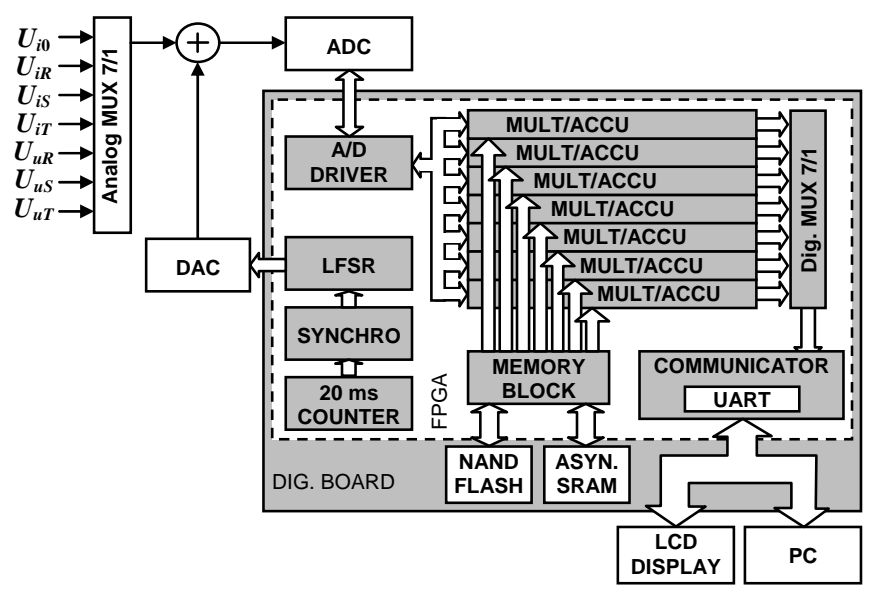

Fig.1. The schematic diagram of the multi-channel instrument for harmonic measurements obtaining coefficients $a_{n}$ and $b_{n}$.

Substituting (3) into (6) and (7), interchanging the order of the sum and the integral and taking into account that $b_{0}$ is identical to zero we can rewrite (8) and (9) as:

$$
\begin{gathered}
\hat{a}_{0}=a_{0}+\sum_{k=1}^{M} a_{k} \frac{2}{T_{0}} \int_{0}^{T_{0}} \cos \frac{2 \pi k t}{T} d t+ \\
+\sum_{k=1}^{M} b_{k} \frac{2}{T_{0}} \int_{0}^{T_{0}} \sin \frac{2 \pi k t}{T} d t \\
\hat{a}_{n}=\sum_{k=1}^{M} a_{k} \frac{2}{T_{0}} \int_{0}^{T_{0}} \cos \frac{2 \pi k t}{T} \cos \frac{2 \pi n t}{T_{0}} d t+, n=1, \ldots, M \\
+\sum_{k=1}^{M} b_{k} \frac{2}{T_{0}} \int_{0}^{T_{0}} \sin \frac{2 \pi k t}{T} \cos \frac{2 \pi n t}{T_{0}} d t \\
\hat{b}_{n}=\sum_{k=1}^{M} a_{k} \frac{2}{T_{0}} \int_{0}^{T_{0}} \cos \frac{2 \pi k t}{T} \sin \frac{2 \pi n t}{T_{0}} d t+, n=1, \ldots, M \\
+\sum_{k=1}^{M} b_{k} \frac{2}{T_{0}} \int_{0}^{T_{0}} \sin \frac{2 \pi k t}{T} \sin \frac{2 \pi n t}{T_{0}} d t
\end{gathered}
$$

To simplify the notation, we will use $\xi$ to denote the relative variation of fundamental frequency as given in (10).

$$
\xi=\frac{\Delta f_{0}}{f_{0}} \neq 0
$$

Equations (10) - (12) can be rewritten as (14) - (16) as follows:

$$
\begin{gathered}
\hat{a}_{0}=a_{0}+\sum_{k=0}^{M}\left(a_{k} C_{k}^{\left(a_{0}\right)}+b_{k} S_{k}^{\left(a_{0}\right)}\right) \\
\hat{a}_{n}=\sum_{k=1}^{M} a_{k} C_{k}^{\left(a_{n}\right)}+b_{k} S_{k}^{\left(a_{n}\right)}, n=1, \ldots, M \\
\hat{b}_{n}=\sum_{k=1}^{M} a_{k} C_{k}^{\left(b_{n}\right)}+b_{k} S_{k}^{\left(b_{n}\right)}, n=1, \ldots, M
\end{gathered}
$$

where the coefficients $C_{k}^{\left(a_{n}\right)}, S_{k}^{\left(a_{n}\right)}, C_{k}^{\left(b_{n}\right)}$ and $S_{k}^{\left(b_{n}\right)}$ are given by $(17)-(20)$ :

$$
\begin{aligned}
& \begin{array}{rlr}
C_{k}^{\left(a_{n}\right)} & =\frac{2}{T_{0}} \int_{0}^{T_{0}} \cos \frac{2 \pi k t}{T} \cos \frac{2 \pi n t}{T_{0}} d t=, \quad \begin{array}{ll}
k=1, \ldots, M \\
n=0, \ldots, M
\end{array} \\
& =\frac{\sin (2 \pi k \xi)}{\pi} \cdot \frac{k(1+\xi)}{k^{2}(1+\xi)^{2}-n^{2}} &
\end{array}
\end{aligned}
$$

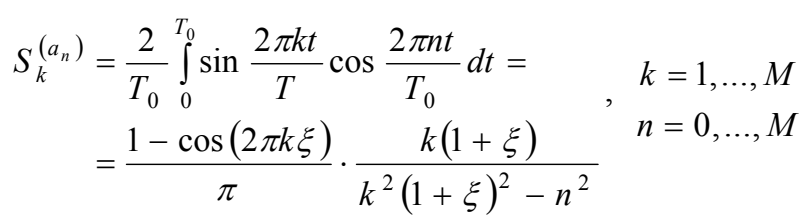

$$
\begin{aligned}
& \begin{array}{rlr}
C_{k}^{\left(b_{n}\right)} & =\frac{2}{T_{0}} \int_{0}^{T_{0}} \cos \frac{2 \pi k t}{T} \sin \frac{2 \pi n t}{T_{0}} d t=\quad, \begin{array}{r}
k=1, \ldots, M \\
n=1, \ldots, M
\end{array} \\
& =-\frac{1-\cos (2 \pi k \xi)}{\pi} \cdot \frac{n}{k^{2}(1+\xi)^{2}-n^{2}} &
\end{array}
\end{aligned}
$$

$$
\begin{array}{rlr}
S_{k}^{\left(b_{n}\right)} & =\frac{2}{T_{0}} \int_{0}^{T_{0}} \sin \frac{2 \pi k t}{T} \sin \frac{2 \pi n t}{T_{0}} d t=, & k=1, \ldots, M \\
& =\frac{\sin (2 \pi k \xi)}{\pi} \cdot \frac{n}{k^{2}(1+\xi)^{2}-n^{2}} &
\end{array}
$$

Relations (14) - (16) can be regarded as a system of equations where $\hat{a}_{n}$ and $\hat{b}_{n}$ are pseudo-harmonics obtained from an incorrect measurement and $a_{n}$ and $b_{n}$ are the correct values, which have to be reconstructed. Notice that $a_{0}$ does not configure in (15) and (16), and therefore the equation (14) can be solved later, when $a_{n}$ and $b_{n}$ are recovered.

Thus, we reduce the problem of the error elimination in harmonic measurement, which is caused by the frequency variation, onto the problem of solving the system of linear equations (15) - (16), which can be represented in the following matrix form

$$
\left[\begin{array}{l}
\hat{a}_{n} \\
\hat{b}_{n}
\end{array}\right]=A\left[\begin{array}{l}
a_{n} \\
b_{n}
\end{array}\right]
$$

where $A$ is the matrix of order $2 M$, given as follows:

$A=\left[\begin{array}{cccc:cccc}C_{1}^{\left(a_{1}\right)} & C_{2}^{\left(a_{1}\right)} & \cdots & C_{M}^{\left(a_{1}\right)} & S_{1}^{\left(a_{1}\right)} & S_{2}^{\left(a_{1}\right)} & \ldots & S_{1}^{\left(a_{1}\right)} \\ C_{1}^{\left(a_{2}\right)} & C_{2}^{\left(a_{2}\right)} & \cdots & C_{M}^{\left(a_{2}\right)} & S_{1}^{\left(a_{2}\right)} & S_{2}^{\left(a_{2}\right)} & \ldots & S_{1}^{\left(a_{2}\right)} \\ \vdots & \vdots & \ddots & \vdots & \vdots & \vdots & \ddots & \vdots \\ C_{1}^{\left(a_{M}\right)} & C^{\left(a_{M}\right)} & \cdots & C_{M}^{\left(a_{M}\right)} & S_{1}^{\left(a_{M}\right)} & S_{2}^{\left(a_{M}\right)} & \ldots & S_{M}^{\left(a_{M}\right)} \\ \hdashline C_{1}^{\left(b_{1}\right)} & C_{2}^{\left(b_{1}\right)} & \cdots & C_{M}^{\left(b_{1}\right)} & S_{1}^{\left(b_{1}\right)} & S_{2}^{\left(b_{1}\right)} & \cdots & S_{M}^{\left(b_{1}\right)} \\ C_{1}^{\left(b_{2}\right)} & C_{2}^{\left(b_{2}\right)} & \cdots & C_{M}^{\left(b_{2}\right)} & S_{1}^{\left(b_{2}\right)} & S_{2}^{\left(b_{2}\right)} & \cdots & S_{M}^{\left(b_{2}\right)} \\ \vdots & \vdots & \ddots & \vdots & \vdots & \vdots & \ddots & \vdots \\ C_{1}^{\left(b_{M}\right)} & C_{2}^{\left(b_{M}\right)} & \cdots & C_{M}^{\left(b_{M}\right)} & S_{1}^{\left(b_{M}\right)} & S_{2}^{\left(b_{M}\right)} & \cdots & S_{M}^{\left(b_{M}\right)}\end{array}\right]$

When matrix $A$ is non-singular, the system (21) has a unique solution for vector $\left[\begin{array}{ll}a_{n} & b_{n}\end{array}\right]^{\mathrm{T}}$. 
To solve (21) $\xi$ must be known. However, $\xi$ is not known in advance, prior to the measurement interval since the actual fundamental frequency $f$ of the measured signal drifts for an unknown amount from the nominal value $f_{0}$ and the instrument does not adjust the frequency of its internal oscillator to this change.

There are methods for the estimation of frequency disturbances in power grids calculated directly from the obtained DFT coefficients, e.g., around component peaks [22]. But such an estimate is based on the DFT coefficients which are inherently erroneous and, thus, it is not suitable for a further correction of the DFT coefficients themselves.

Nevertheless, the value for $\xi$ can be determined independently and in parallel with the pseudo-harmonic measurement using a simple frequency extraction circuit shown in Fig. 2 and made available at the end of each measurement interval.

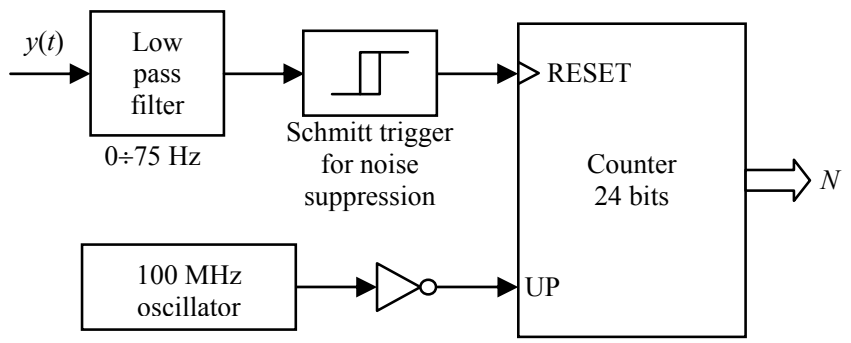

Fig.2. The schematic diagram of the frequency extraction circuit for obtaining the parameter $\xi$.

Using the circuitry in Fig.2 $\xi$ can be estimated as

$$
\hat{\xi}=\frac{100 \mathrm{MHz}}{N \cdot f_{0}}-1
$$

where $N$ is the output of the counter in Fig.2.

Let $N_{0}$ denote the output of the counter when the actual frequency equals the nominal value $\left(f=f_{0}\right)$. For $N_{0}$ the parameter $\hat{\xi}$ equals zero. The minimal value of $\hat{\xi}$ is thus obtained for

$$
N=N_{0} \pm 1
$$

and it equals $\pm 0.5 \times 10^{-6}$ for $f_{0}=50 \mathrm{~Hz}$ and $\pm 0.6 \times 10^{-6}$ for $f_{0}=60 \mathrm{~Hz}$. It will be shown later in this paper that these values satisfy all practical cases of interest.

The estimate for $\xi$ obtained from (23) is independent from harmonic measurements and it is available after each measurement interval, e.g., signal period. It can be therefore considered a known parameter available for the population of the matrix $A$ and the correction of the measurement results by solving (21).

The following number of multiplications and additions is required to create the matrix $A$ in the case when coefficients given by (17) - (20) are calculated using Taylor's polynomials of $10^{\text {th }}$ order:

$$
75 \cdot(2 M)(2 M+1)+2 M, \text { multiplications and }
$$

$$
13 \cdot(2 M)(2 M+1), \text { additions }
$$

For example, when $M=50$ the number of multiplications is 757700 and the number of additions is 131300 .

Further, we will provide conditions in which $A$ can be nonsingular. Moreover, we will show that under certain conditions this matrix is diagonally dominant, which allows us to use an extremely efficient tool for solving (21). Unlike the general purpose algorithms, such as Gaussian elimination, the procedure based on diagonally dominant matrices (DDM) has a fast convergence, it is not sensitive to small coefficient variations, does not accumulate numerical error, does not waste time on performing the column permutation and does not require any decision making or branching. This means that it can be efficiently applied for on-the-fly correction of the measurement results using minimal computational resources.

\section{Diagonally DOMINANT MATRICES}

A square matrix $X=\left[x_{i j}\right]_{N \times N}$ is said to be a diagonally dominant matrix (DDM) if its elements satisfy the condition (27).

$$
\left|x_{i i}\right| \geq \sum_{\substack{j=1 \\ j \neq i}}^{N}\left|x_{i j}\right|, \quad i=1 . . N
$$

An important feature of the DDM is its non-singularity. For solving the system of linear equations involving a DDM, the Jacobi iterative method can be used [23].

For a single iteration in a system of order $100(M=50$ harmonics) the Jacobi method requires

$$
(2 M)^{2}=10000 \text { multiplications and }
$$

$$
(2 M)^{2}=10000 \text { additions }
$$

For the worst case of the parameter $\xi$, when the matrix $A$ is at the edge of being a DDM, and depending on the input signal composition, it may take up to 500 iterations to reach the error of $0.01 \%$, which is at least 10 times lower than the common harmonic measurement uncertainty of commercial power quality analyzers. This number of iterations can be achieved even with an embedded PC with modest 1 GFlops. Such a device can ensure at least 750 iterations within a single period of the input signal $(20.0 \mathrm{~ms}$ or $16.7 \mathrm{~ms})$. In practical cases of interest the number of iterations is usually much smaller and far below 100 .

For comparison, in Table 1 we provide an estimate of the time required for the algorithm to execute on various platforms in 32-bit arithmetic under the most extreme conditions: $M=100$ or 80,000 floating point operations and assuming maximum 500 iterations [24], [25], [26], [27], [28], [29]. We have considered platforms suitable for both mainframe (high performance) and for terrain instruments (low GFlops/watt ratio). However, the values in Table 1 do not include times required for memory fetch operations due to the complexity of the issue across different platforms and their dependency on the BUS realization. 
Table 1. Estimates of algorithm duration on various platforms, for correcting 100 harmonic values in 500 iterations

\begin{tabular}{|l|c|r|r|}
\hline \hline \multicolumn{1}{|c|}{ Platform } & Type & GFlops & $\begin{array}{c}\text { Estimate of } \\
\text { algorithm } \\
\text { duration }\end{array}$ \\
\hline Intel i5-3570T & $\mu \mathrm{P}$ & 106 & $377 \mu \mathrm{s}$ \\
\hline Intel i7-990x & $\mu \mathrm{P}$ & 90 & $444 \mu \mathrm{s}$ \\
\hline Intel Core i7-3770 & mobile $\mu \mathrm{P}$ & 225 & $178 \mu \mathrm{s}$ \\
\hline Intel Core Duo L7700 & mobile $\mu \mathrm{P}$ & 14.4 & $2778 \mu \mathrm{s}$ \\
\hline AMD Opteron 6284 SE & embedded $\mu \mathrm{P}$ & 346 & $116 \mu \mathrm{s}$ \\
\hline AMD Radeon HD & mobile $\mu \mathrm{P}$ & 4300 & $9 \mu \mathrm{s}$ \\
\hline TI Sitara Cortex-A8 & $\mu \mathrm{P}$ & 4 & $10,000 \mu \mathrm{s}$ \\
\hline TI TMS320 C6655 & DSP & 165 & $242 \mu \mathrm{s}$ \\
\hline NVidia GTX 690 & mobile $\mu \mathrm{P}$ & 5621 & $7 \mu \mathrm{s}$ \\
\hline NVidia Tesla K10 & mobile $\mu \mathrm{P}$ & 4577 & $9 \mu \mathrm{s}$ \\
\hline IBM Power A2 & mobile $\mu \mathrm{P}$ & 204 & $196 \mu \mathrm{s}$ \\
\hline Altera Stratix IV & FPGA & 171 & $234 \mu \mathrm{s}$ \\
\hline Altera Stratix V & FPGA & 1000 & $40 \mu \mathrm{s}$ \\
\hline Adapteva Epiphany-IV & mobile $\mu \mathrm{P}$ & 100 & $400 \mu \mathrm{s}$ \\
\hline \hline
\end{tabular}

Table 2. Maximum allowed values for frequency drift $\xi$ for obtaining diagonally dominant and/or non-singular matrix $A$ depending on the number of harmonics $M$ measured

\begin{tabular}{|c|c|c|c|c|}
\hline \hline \multirow{2}{*}{$M$} & \multicolumn{4}{|c|}{$\xi_{\text {max }}$} \\
\cline { 2 - 5 } & $\begin{array}{c}\xi>0 \\
\text { is DDM }\end{array}$ & $\begin{array}{c}\xi<0 \\
A \text { is DDM }\end{array}$ & $\begin{array}{c}\xi>0 \\
A \text { is non- } \\
\text { singular }\end{array}$ & $\begin{array}{c}\xi<0 \\
A \text { is non- } \\
\text { singular }\end{array}$ \\
\hline 1 & 0.250000 & -0.250000 & 1.000000 & -0.999999 \\
\hline 2 & 0.101882 & -0.105962 & 0.500000 & -0.999999 \\
\hline 5 & 0.033161 & -0.035227 & 0.200000 & -0.999999 \\
\hline 10 & 0.014307 & -0.014257 & 0.100000 & -0.999999 \\
\hline 15 & 0.008649 & -0.008684 & 0.066667 & -0.999999 \\
\hline 20 & 0.006107 & -0.006094 & 0.050000 & -0.999999 \\
\hline 25 & 0.004645 & -0.004646 & 0.040000 & -0.999999 \\
\hline 30 & 0.003731 & -0.003730 & 0.033333 & -0.999999 \\
\hline 35 & 0.003097 & -0.003095 & 0.028571 & -0.999999 \\
\hline 40 & 0.002638 & -0.002639 & 0.025000 & -0.999999 \\
\hline 45 & 0.002293 & -0.002291 & 0.022222 & -0.999999 \\
\hline 50 & 0.002021 & -0.002020 & 0.020000 & -0.999999 \\
\hline 55 & 0.001805 & -0.001805 & 0.018182 & -0.999999 \\
\hline 60 & 0.001628 & -0.001627 & 0.016667 & -0.999999 \\
\hline 65 & 0.001480 & -0.001480 & 0.015384 & -0.999999 \\
\hline 70 & 0.001356 & -0.001356 & 0.014286 & -0.999999 \\
\hline 75 & 0.001250 & -0.001250 & 0.013333 & -0.999999 \\
\hline 80 & 0.001158 & -0.001158 & 0.012500 & -0.999999 \\
\hline 85 & 0.001079 & -0.001079 & 0.011765 & -0.999999 \\
\hline 90 & 0.001009 & -0.001008 & 0.011111 & -0.999999 \\
\hline 95 & 0.000946 & -0.000946 & 0.010526 & -0.999999 \\
\hline 100 & 0.000891 & -0.000891 & 0.010000 & -0.999999 \\
\hline & & & & \\
\hline
\end{tabular}

It is evident from Table 1 that the state-of-the-art technology allows for an easy real time implementation of the proposed algorithm regardless of the considered platform ( $\mu \mathrm{P}$, mobile $\mu \mathrm{P}$, embedded $\mu \mathrm{P}$, DSP, FPGA etc.)

Further, we will discuss the conditions under which the matrix $A$, whose inverse matrix provides correction of the measurement results, is diagonally dominant. This is of special interest in cases where modest computational resources need to achieve high performance corrections.

The elements of $A$ are all functions of the parameter $\xi$. For $\xi=0$, the matrix $A$ equals the identity matrix $E=\operatorname{diag}(1,1, \ldots, 1)$ and the condition $(24)$ is satisfied.
As $|\xi|$ becomes greater than zero, absolute values of the elements on the main diagonal decrease, remaining close to $1 \mathrm{~s}$, while absolute values of other elements slightly increase. Thus, the matrix $A$ disperses from identity matrix to a diagonally dominant matrix. Four cases of 100x100 matrices $A$ defined with (22) for different values of parameter $\xi$ are illustrated in Fig.3.

Each pixel in Fig. 3 represents one coefficient of the matrix $A$. Dark tones indicate higher values of the elements, while pale tones indicate smaller values. Black equals to the maximum absolute value \pm 1 and white equals to 0 .

A computer simulation was used to sweep the space of possible values for $\xi$ in order to find positive and negative limit values for which the matrix $A$ is the DDM depending on the number of harmonics $M$. This implies that between these limit values matrix $A$ is non-singular. We have also explored the conditions under which the matrix $A$ is nonsingular beyond the interval of diagonal dominancy. The results of the simulation are presented in Fig.4 and in Table 2.

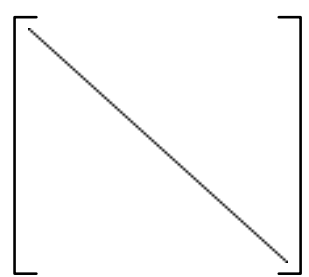

$\xi=0$

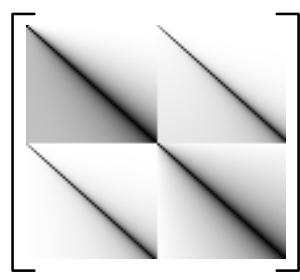

$\xi= \pm 0.0002$

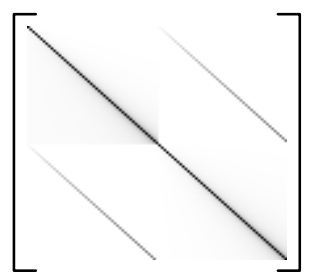

$\xi= \pm 0.0001$

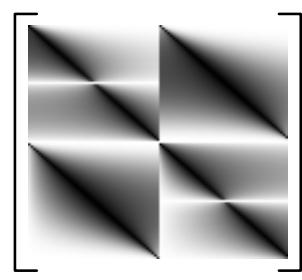

$\xi= \pm 0.002$
Fig.3. Several examples of diagonally dominant matrices $A$ of order $100 \times 100$ for measuring up to $M=50$ harmonics

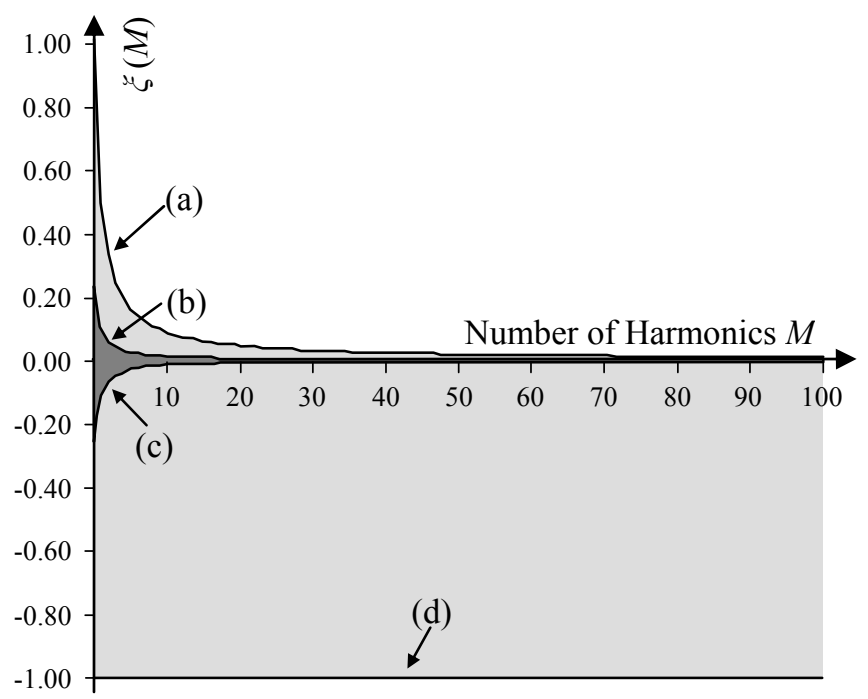

Fig.4. Zones of diagonal dominance and of non-singularity of matrix $A$ for various combinations of $M$ and $\xi$ 
The matrix $A$ is diagonally dominant if $\xi$ is within the dark-grey zone in Fig.4, i.e. between the limit curves (b) and (c). This zone will be called the DDM zone. The matrix $A$ is non-singular if $\xi$ is between the limit curves (a) and (d), i.e. in the union of light-grey and dark-grey zones. We will call it the Non-singularity zone. For the remaining pairs $(\xi, M)$ belonging to the white region, which will be named the Singularity zone, the measurement results cannot be corrected.

Computer simulation suggests that the limit curve (a) in Fig.4 for positive values of $\xi$ can be approximated well enough by hyperbole $1 / M$. Thus, the region or nonsingularity for positive $\xi$ can be described by the following relation:

$$
\xi \cdot M \leq 1
$$

Notice that for negative values of $\xi$ the matrix $A$ is nonsingular for every $(\xi, M)$ in contrast to the situation when $\xi>0$. This can easily be explained by taking into account that for a negative frequency drift of the fundamental frequency all the harmonics shift towards lower frequencies. Thus, the measured pseudo-harmonics contain all the necessary information for the reconstruction of the actual spectrum. In contrast to that, a positive frequency drift can cause the spectrum to slide outside the measurement region $\left(M \cdot f_{0}\right)$ and the information about higher order harmonics will be lost. When (30) is no longer satisfied, more than $M$ harmonics are required to reconstruct the actual spectrum of the measured input signal, e.g., $M+k$. However, $k$ is an unknown number since the parameter $\xi$ is not known in advance. Therefore, such situation is of no practical interest and we will consider that (21) cannot be solved in that case.

\section{DISCUSSION ON THE RANGE OF APPLICABILITY OF THE PROPOSED METHOD}

Concerning the DDM zone in Fig.4, it may seem discouraging that it is rather narrow. Nevertheless, it turns out that the DDM zone covers most of the cases of practical interest. The following discussion proves this statement. Should one want to measure 50 harmonics and apply the correction method using the Jacobi iterative method, e.g., in a European power grid, having the fundamental frequency of $50 \mathrm{~Hz}$, according to (30), the parameter $\xi$ has to lay approximately within the interval $\pm 0.002( \pm 0.2 \%)$. This interval for $\xi$ corresponds to an absolute frequency drift of $\pm 0.1 \mathrm{~Hz}$, which is hardly to be reached even over a longer period.

Frequency drifts beyond $\pm 0.1 \mathrm{~Hz}$ may occur, but they are commonly associated to abrupt changes in power consumption (up to 10 megawatts) lasting for a rather small number of periods [7]. Prolonged frequency excursions commonly lead to power failures where different measurement procedures must be applied. In stabilized power grids these frequency excursions have a very short occurrence as it is noticed in [30] and [31].

Smaller and shorter discrepancies of the frequency can be assessed directly [10], [32] and can be used in perturbation calculus to estimate the scope of the fundamental frequency excursion and its influence on the accuracy of harmonic measurements.
Measurement results performed in power grid in Serbia prior to and upon the interconnection with the European power system given in [33] demonstrate that the frequency is stable enough to guarantee that the matrix $A$ is the DDM in the majority of harmonic measurement applications, especially where an averaging over significant number of periods is performed.

Even in cases when the matrix $A$ is not strictly a DDM but is non-singular (the light-grey zone), according to Fig.4 a reduction of the number of harmonics $M$ could relocate the pair $(\xi, M)$ within the DDM zone. For example, if the initial request is to measure 65 harmonics under the fundamental frequency drift of $\pm 0.08 \mathrm{~Hz}(\xi= \pm 0.0016)$, a reduction of the number of harmonics from 65 to 60 is sufficient to ensure that matrix $A$ is the DDM.

A strong point of the proposed method is that it requires relative frequency changes to be within the interval given with (30), and not just an absolute change around $50 \mathrm{~Hz}$ or $60 \mathrm{~Hz}$. This is of interest in cases when an arbitrary bias in frequency is present and variations occur around a new central frequency. Even within the instruments that can adapt to such new central frequency, the proposed method allows for a correction of measurement results. The correction will always be necessary since the adaptation can only be achieved with a finite resolution. A frequency extraction circuit such as PLL can be used to come as close to the actual fundamental frequency as possible and leave out a very small difference between the presumed and actual frequency [11]. The method described in [11] can also be used to by-pass the correction post-processing of the measurement results, but the synchronization circuits in that case have to be extremely fast.

\section{EXPERIMENTAL RESULTS}

A prototype instrument has been made, described in Error! Reference source not found. shown in Fig.5. The instrument measures 3 voltage and 4 current signals and for each obtains coefficients of the trigonometric polynomial representation of order 50 with a 16-bit resolution within one period of the fundamental frequency. Thus, the instrument produces 2 Bytes x 100 coefficients $x 7$ channels $\mathrm{x} 50$ signal periods $=70 \mathrm{kB}$ of measurement data per second. It is primarily used for measurements of the power grid integral parameters such as active, reactive and apparent power. However, the measurement data rate indicates that a similar method can also be applied in diagnostic instruments such as fault recorders.

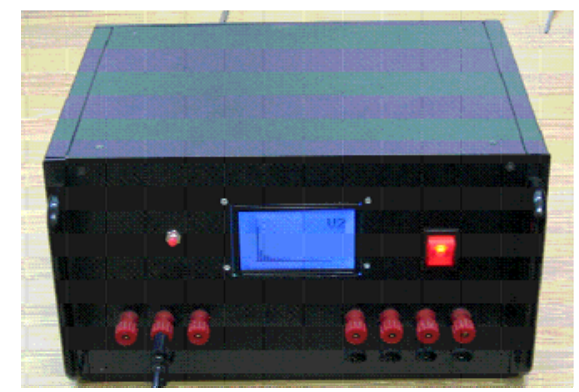

Fig.5. Integrated stochastic instrument with seven channels 1-channel instr. with referent input signal 


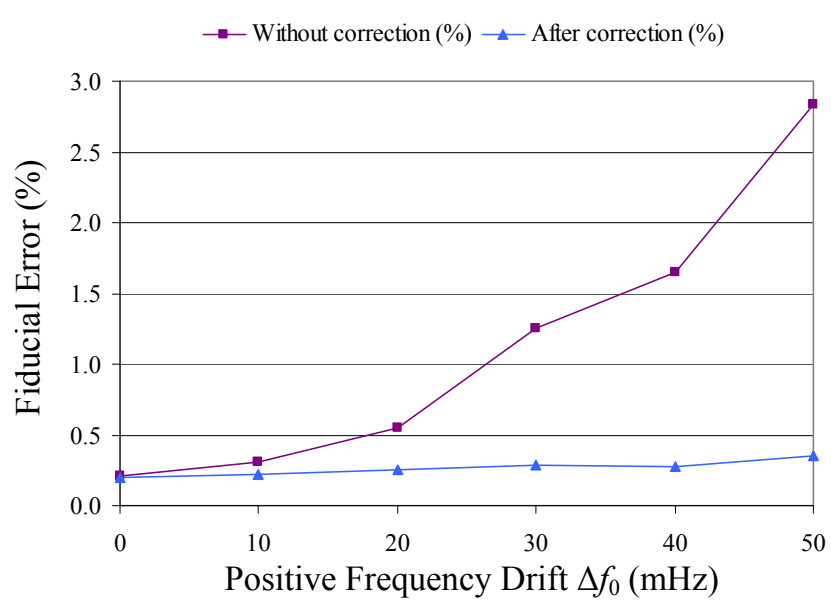

Fig.6. Measurement error of 1-channel stochastic instruments with and without the correction method

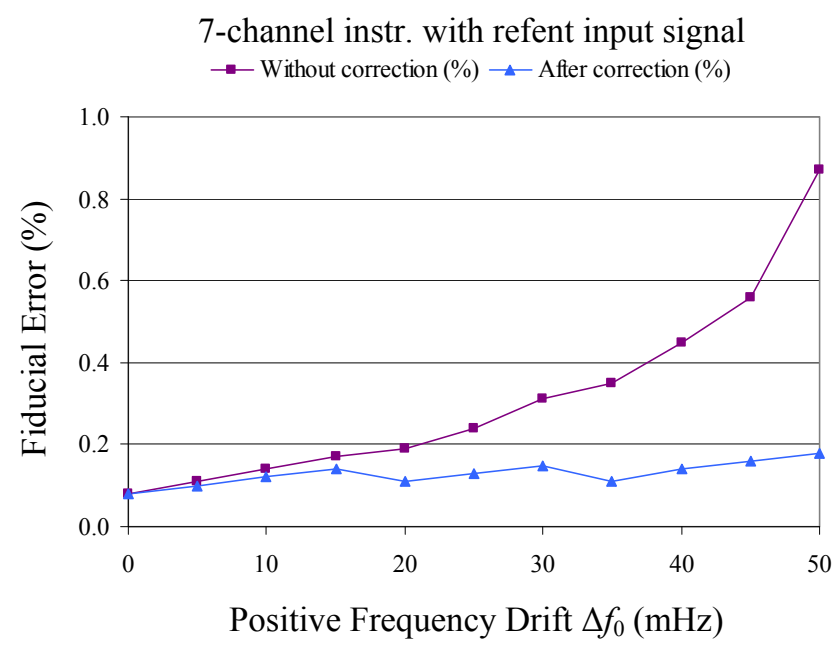

Fig.7. Measurement error of 7-channel stochastic instruments with and without the correction method

7-channel instr. with rectangular input signal

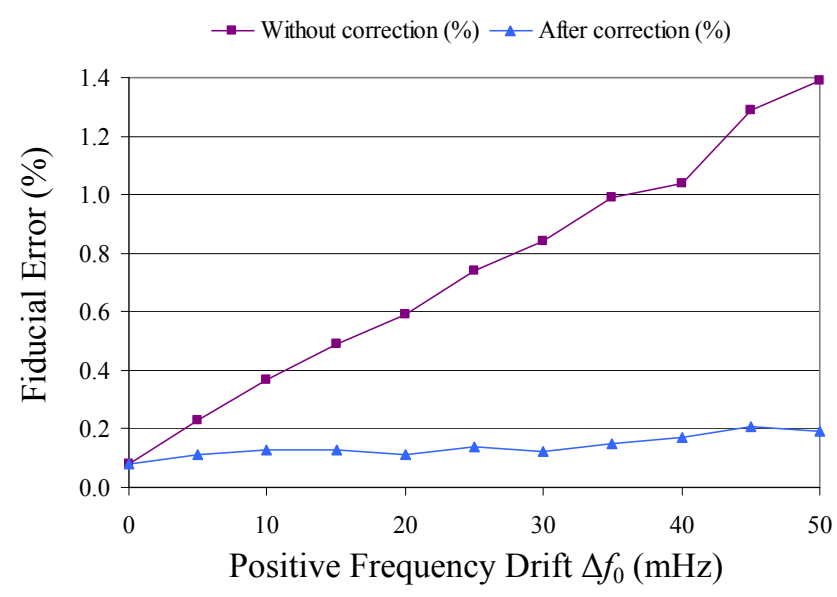

Fig. 8. Measurement error of 7-channel stochastic instruments with and without the correction calculus for a rectangular input signal
The operation of the instrument is, however, based on an assumption that the fundamental frequency in power grid is always equal to nominal $(50 \mathrm{~Hz}$ or $60 \mathrm{~Hz})$.

The authors have applied the proposed method to a 1-channel integrated stochastic instrument for measuring up to 16 harmonics described in [34] and to a 7-channel integrated stochastic instrument for measuring up to 50 harmonics described in [16]. Both instruments have a unique ability to measure phase and amplitude of a harmonic with an arbitrary small error that is independent on the order of the harmonic or the waveform of the input signal [16].

The calibration and measurements have been performed in the Laboratory for Electrical Quantities at the Faculty of Technical Sciences in Novi Sad, Serbia. The measurement results for the 1-channel instrument are given in Fig.6. The measurement results for the 7-channel instrument are given in Fig. 7 and Fig.8.

The results in Fig. 6 to Fig. 8 have been obtained using 100 iterations. Since the error is independent of the order of the harmonic, only one resulting graph is given for all values of $M$ up to 50. The fiducial error on the vertical scale is the ratio of the absolute error to the instrument's full scale. The fiducial error is used because the same ADC is applied for measuring instances of two different physical quantities voltage and current. Since an input to the ADC can only be voltage (Fig.1), each current is being transferred into the corresponding voltage using a transresistance circuit. Thus the use of the fiducial error allowed for a more uniform characterization of the instrument's measurement uncertainty across all seven parameters of interest.

After applying the proposed correction method in both 1-channel and 7-channel instrument, the measurement uncertainty has become considerably less dependent on frequency variations below the criterion given with (30).

\section{CONCLUSION}

A new method has been presented for on-the-fly correction of the measurement results of voltage and current harmonics in power grids due to the difference between the signal actual frequency and the locally assumed reference frequency within the instrument with an internal oscillator.

The idea behind the correction method is based on the fact that the matrix which maps the correct values into incorrect measurement results has a tendency to cumulate around the main diagonal. This fact allows for an application of an efficient algorithm for elimination of measurement error caused by small frequency drifts that are present in real power grid signals. The domain of applicability of the method is presented and widely discussed in this paper.

The authors provided the conditions for the correction matrix to be non-singular beyond the interval of diagonal dominancy including modification procedures, which guarantee diagonal dominancy on the expense of reducing the number of required harmonics.

The method proves to be equally significant both for instruments which can and cannot adapt to frequency changes as discussed in this paper. The application of the proposed method requires modest computational and hardware resources, thus it can be easily implemented in new measurement equipment, as well as in most of the 
existing instruments for power quality measurements offering lower measurement uncertainty.

The experimental results showed that by applying the proposed method, the fiducial error is substantially lowered and almost independent on the frequency drift providing that the conditions considered in this paper are met.

\section{ACKNOWLEDGMENT}

This work was supported by the Ministry of Education and Science of the Republic of Serbia under research grants Nos. TR32019 and III43011 started on 01.01.2011.

\section{REFERENCES}

[1] Stones, J., Collinson, A. (2001). Power quality. Power Engineering Journal, 15 (2), 58-64.

[2] Ghandehari, R., Jalilian, A. (2004). Economical impacts of power quality in power systems. In 39th International Universities Power Engineering Conference (UPEC 2004), 6-8 September 2004. IEEE, Vol. 2, 893-897.

[3] Thapar, A., Saha, T.K., Zhao, Y.D. (2004). Investigation of power quality categorisation and simulating its impact on sensitive electronic equipment. In IEEE Power Engineering Society General Meeting, 6-10 June 2004. IEEE, Vol. 1, 528-533.

[4] Sermon, R.C. (2005). An overview of power quality standards and guidelines from the end-user's point-ofview. In Rural Electric Power Conference, 8-10 May 2005. IEEE, B1/1-B1/5.

[5] Broshi, A. (2007). Monitoring power quality beyond EN 50160 and IEC 61000-4-30. In 9th International Conference on Electrical Power Quality and Utilisation (EPQU 2007), 9-11 October 2007. IEEE, 1-6.

[6] Kuhlmann, V., Sinton, A., Dewe, M., Arnold, C. (2007). Effects of sampling rate and ADC width on the accuracy of amplitude and phase measurements in power-quality monitoring. IEEE Transactions on Power Delivery, 22 (2), 758-764.

[7] European Committee for Standardization. (1999). Voltage Characteristics of Electricity Supplied by Public Distribution Systems. European Standard EN 50160.

[8] Mandache, L., Al-Haddad, K. (2005). New high precision harmonic analysis method for power quality assessment. In Canadian Conference on Electrical and Computer Engineering (CCECE 2005), 1-4 May 2005. IEEE, 1958-1961.

[9] Szmajda, M., Gorecki, K., Mroczka, J. (2007). DFT algorithm analysis in low-cost power quality measurement systems based on a DSP processor. In 9th International Conference on Electrical Power Quality and Utilisation (EPQU 2007), 9-11 October 2007. IEEE, 1-6.

[10] Moo, C.S., Chang, Y.N., Mok, P.P. (1995). A digital measurement scheme for time-varying transient harmonics. IEEE Transactions on Power Delivery, 10 (2), 588-594.
[11] Karimi-Ghartemani, M., Iravani, M.R. (2005). Measurement of harmonics/inter-harmonics of timevarying frequencies. IEEE Transactions on Power Delivery, 20 (1), 23-31.

[12] Yoon, W.K., Devaney, M.J. (2000). Reactive power measurement using the wavelet transform. IEEE Transactions on Instrumentation and Measurement, 49 (2), 246-252.

[13] Espel, P., Poletaeff, A., Ndilimabaka, H. (2010). Traceability of voltage measurements for nonsinusoidal waveforms. Measurement Science Review, 10 (6), 200-204.

[14] Vujicic, V.V., Milovancev, S.S., Pesaljevic, M.D., Pejic, D.V., Zupunski, I.Z. (1999). Low-frequency stochastic true RMS instrument. IEEE Transactions on Instrumentation and Measurement, 48 (2), 467-470.

[15] Vujicic, V.V. (2001). Generalized low frequency stochastic true RMS instrument. IEEE Transactions on Instrumentation and Measurement, 50, 1089-1092.

[16] Pjevalica, V., Vujicic, V. (2005). Further generalization of the low-frequency true-RMS instrument. In Proceedings of the IEEE Instrumentation and Measurement Technology Conference, May 2005. IEEE, 1008-1011.

[17] Weierstrass, K. (1885). Über die analytische Darstellbarkeit sogenannter willkürlicher Functionen einer reellen Veränderlichen. In Sitzungsberichte der Königlich Preußischen Akademie der Wissenschaften zu Berlin.

[18] Stone, M.H. (1948). The generalized Weierstrass approximation theorem. Mathematics Magazine, 21 (4), 167-184; 21 (5), 237-254.

[19] Kamenský, M., Kováč, K. (2011). Correction of ADC errors by additive iterative method with dithering. Measurement Science Review, 11 (1), 15-18.

[20] Hegeduš, H., Mostarac, P., Malarić, R. (2011). Comparison of RMS value measurement algorithms of non-coherent sampled signals. Measurement Science Review, 11 (3), 79-84.

[21] Antic, B., Pjevalica, N. (2006). A new approach to power grid measurements - measuring in frequency domain. In Second Regional Conference and Exhibition on Electricity Distribution, October 17-20 2006. Book of Abstracts, 37-38.

[22] Agrež, D. (2010). Estimation and tracking of the power quality disturbances in the frequency domain. Measurement Science Review, 10 (6), 189-194.

[23] Acton, F.S. (1990). Numerical Methods that Work (2nd ed.). Washington, DC: Mathematical Association of America, 161-163.

[24] Intel Corporation. (2012). Support for processors. $\mathrm{http} / / / \mathrm{www}$. intel.com/p/en_US/support/processors

[25] Advanced Micro Devices, Inc. (2012). Products and technologies. http://www.amd.com/us/products/ Pages/products.aspx

[26] Altera Corporation. (2011). Altera floating point megafunctions. http:/www.altera.com/literature/ug/ ug_altfp_mfug.pdf 
[27] Advanced Micro Devices, Inc. (2012). AMD opteron processors comparison. http://sites.amd.com/us/ Documents/49747D_HPC_Processor_Comparison_v3 July2012.pdf

[28] Texas Instruments. (2012). Embedded processors. http://www.ti.com/lsds/ti/dsp/embedded_ processor.page

[29] Hindriksen, V. (2012). Processors that can do 20+ GFLOPS per Watt. http://www.streamcomputing.eu/ blog/2012-08-27/processors-that-can-do-20-gflopswatt/

[30] Kundur, P., Paserba, J., Ajjarapu, V., et al. (2004). Definition and classification of power system stability IEEE/CIGRE joint task force on stability terms and definitions. IEEE Transactions on Power Systems, 19 (2), 1387-1401.

[31] Horne, J., Flynn, D., Littler, T. (2004). Frequency stability issues for islanded power systems. In IEEE PES Power Systems Conference and Exposition, 10-13 October 2004. IEEE, Vol. 1, 299-306.
[32] Cai, Z.X., Ni, Y.X. (2000). A direct method for frequency stability assessment of power systems. In International Conference on Advances in Power System Control, Operation and Management (APSCOM-00), 30 October - 1 November 2000. IEEE, Vol. 1, 285-289.

[33] Antic, B.M., Vujicic, V.V. (2007). An improved version of the stochastic harmonic instrument. In Proceedings of the Metrology Congress 2007, 26-28 September 2007.

[34] Tomic, J. (2004). Integrated Instrument for Harmonics Measurement. Unpublished M.Sc. thesis, University of Novi Sad, Faculty of Technical Sciences, Novi Sad, Serbia.

Received February 13, 2012. Accepted November 20, 2012. 